ISSN 1997-5902

\title{
Inventaire floristique et écologie des macrophytes aquatiques de la rivière Kambo à Douala (Cameroun)
}

Siegfried Didier DIBONG1,2, 3 , Gildas Parfait NDJOUONDO*1

(1) Département de Biologie des Organismes Végétaux, Faculté des Sciences, Université de Douala, B.P. 24157 Douala, Cameroun

(2) Département des Sciences Pharmaceutiques, Faculté de Médecine et des Sciences Pharmaceutiques, B.P. 2701 Douala, Cameroun

(3) Département d'Aquaculture, Institut des Sciences Halieutiques, B.P. 2701 Douala, Cameroun

*Auteur de la correspondance : parfaitgildas@yahoo.fr

Original submitted in on $16^{\text {th }}$ May 2014. Published online at www.m.elewa.org on $31^{\text {st }}$ August 2014. http://dx.doi.org/10.4314/jab.v80i1.15

\section{RÉSUMÉ}

Objectif : L'anthropisation galopante associée à la prolifération anarchique des industries et de l'agriculture, dans les bas fonds prédisposent la ville de Douala à une dégradation des zones humides côtières et continentales. L'objectif de l'étude a été de montrer l'influence des facteurs physico-chimiques sur la distribution des macrophytes de la rivière Kambo.

Méthodologie et Résultats : Des inventaires floristiques basés sur l'élaboration des transects ont permis de confectionner des relevés d'espèces à partir des coefficients d'abondance-dominance de Braun-Blanquet et de sociabilité. Des paramètres physico-chimiques de la rivière Kambo ont été pris et mesurés. La répartition des macrophytes varie avec l'indice de diversité de Shannon qui décroît de la station 1 (amont) $(2,96)$ vers la station 3 (aval) $(1,76$ ). La station 3 (aval) a montré une évolution progressive des strates herbacées à la strate arbustive à Alchornea cordifolia ayant un indice de diversité faible $(1,47)$. Le $\mathrm{pH}$, la conductivité et le pourcentage du substrat de fond ont montré que le sol est non salin et vaseux dans toutes les stations, neutre à l'aval et légèrement acide en amont. Onze groupements végétaux ont été identifiés avec deux lots corrélés au stade d'évolution de la végétation, le premier constitué des groupements 1, 2, 3, 4, 5, 6 et 8 appartenant aux stations 1 et 2 et correspondant aux strates herbacées et le second constitué des groupements 9,10 et 11 appartenant à la station 3 et correspondant aux strates à la fois herbacée et arbustive.

Conclusion et application des résultats : Les macrophytes de la rivière Kambo montrent une répartition de la végétation qui est fonction de la composition floristique d'une part et d'autre part des activités anthropiques (milieux dégradé et milieux non dégradés). L'étude écologique réalisée permet de maîtriser les facteurs abiotiques et biotiques qui président à la gestion et la conservation des zones humides côtières, pour le développement durable et le bien être des populations impliquées.

Mots clés : Macrophytes, relevés floristiques, groupement, station, Kambo, Douala

Objective: The rampant human impacts associated with uncontrolled proliferation of industries and agriculture in the lowlands predisposes Douala to degradation of coastal and inland wetlands. The objective of the study was to show the influence of physicochemical factors on the distribution of macrophytes of Kambo river. Methodology and Results: floristic inventories based on the development of transects helped to make surveys by using coefficients of abundance-dominance and sociability. Physico-chemical parameters of the river Kambo 
were taken and measured. The distribution of macrophytes varies with Shannon diversity index, decreasing from station 1 (upstream) (2.96) to station 3 (downstream) (1.76). Station 3 (downstream) showed a gradual evolution of herbaceous strata to ligneous strata of Alchornea cordifolia with a low diversity index (1.47). PH, conductivity and percentage of bottom substrate showed that the soil is saline and not muddy at all stations, neutral to slightly acidic upstream to downstream. Eleven plant communities were identified with two batches correlated to the evolution of the vegetation stage, the first consisting of groups 1, 2, 3, 4, 5, 6 and 8 belonging to stations 1 and 2 and corresponding to herbaceous strata and second consisting of groups 9,10 and 11 belonging to the station 3 and corresponding to herbaceous and ligneous strata.

Conclusion and application of results: The macrophytes of river Kambo show vegetation distribution which is a function of the floristic composition and anthropogenic activities (Degraded and non degraded environments). The environmental study will control abiotic and biotic factors that govern the management and conservation of coastal wetlands for sustainable development and well being of the people involved. The environmental study will control abiotic and biotic factors that govern the management and conservation of coastal wetlands for sustainable development and well being of the people involved.

Keywords: macrophytes, surveys, group, station, Kambo, Douala

\section{INTRODUCTION}

La ville de Douala est un lieu de concentrations humaines où les impératifs liés au développement de l'agriculture menacent l'équilibre des petits écosystèmes fluviaux côtiers (Bemmo et al., 1998). Cette ville est drainée par de nombreux cours d'eau côtiers (Fogwé et Tchotsoua, 2007). Elle est connue depuis plusieurs décennies pour sa forte productivité industrielle et sa commercialisation agricole (industrie et élevage (ovins et porcins)) (Folack et al., 1999). Or, la production de ces ressources implique une utilisation de produits phytosanitaires (engrais, pesticides) qui pourraient avoir des conséquences sur la qualité de l'eau, et conduire à des changements profonds de la composition et de la structure des peuplements des organismes des hydrosystèmes (Priso et al., 2010). La prise en compte des altérations provoquées par les activités humaines sur ces cours d'eau apparaît actuellement comme une préoccupation majeure. La pollution en milieu urbain peut être déterminée par l'analyse des polluants, par des méthodes satellitaires ou l'analyse des communautés végétales (Priso et al., 2010). Toutefois, nonobstant la pertinence des méthodes analytiques, elles restent de peu d'intérêts dans la détection des pollutions épisodiques (Ramade, 1987). Aussi, les plantes, intégrateurs des phénomènes que connaissent les écosystèmes apparaissent comme des marqueurs des changements environnementaux (Sauberer et al., 2003). L'analyse biologique permet d'identifier les perturbations et leurs effets sur les communautés animales et végétales en place. Aussi, la surveillance de la qualité de l'eau a longtemps été basée sur les analyses physico-chimiques afin de mettre en évidence des pollutions (Buchez et al., 2010). Cependant, celles-ci montrent quelques insuffisances dans la mesure où l'équilibre du milieu aquatique peut être sérieusement affecté lors des apports de polluants massifs mais de courte durée. II est alors improbable de les détecter en échantillonnant l'eau une ou deux fois par mois (Priso et al., 2012). Par ailleurs, les travaux sur la région de Douala restent épairs et embryonnaires (Folack et al., 1999 ; Fogwé et Tchotsoua, 2007 ; Meva'a et al., 2010 ; Priso et al. (2012). II apparaît difficile dans ce contexte de définir dans l'état actuel des connaissances, les caractéristiques générales propres aux macrophytes aquatiques de la rivière Kambo de Douala. L'objectif général de l'étude est de montrer l'influence des facteurs physicochimiques sur la distribution des macrophytes aquatiques de la rivière Kambo. Les objectifs spécifiques sont: (1) de mesurer les paramètres physico-chimiques de la rivière, en fonction de la toposéquence ; (2) d'établir les relevés floristiques et (3) d'en dégager les groupements végétaux. 


\section{MATÉRIEL ET MÉTHODES}

Site d'étude: Douala, ville cosmopolite, capitale économique du Cameroun est située au fond du Golfe de Guinée et traversée par le fleuve Wouri. Son réseau hydrographique est relativement dense, parsemé des zones marécageuses (Fogwé et Tchotsoua, 2007). C'est la ville la plus peuplée de la zone CEMAC avec plus de 2,5 millions d'habitants pour une densité de 9 523,8 $\mathrm{hab} . / \mathrm{km}^{2}$. Elle s'étale sur une superficie de près de 5000 ha en perpétuelle croissance au même titre que sa population (Tatso, 2011). La ville de Douala est subdivisée en 11 sous bassins versants (Meva'a et al., 2010). Le sous bassin versant de Kambo fait partie de la zone à climat équatorial côtier nord. La température moyenne mensuelle varie entre $24,8^{\circ} \mathrm{C}$ de juillet à août et $27,7^{\circ} \mathrm{C}$ en février avec une moyenne annuelle de 26,4 ${ }^{\circ} \mathrm{C}$. Les précipitations montrent que Douala est pluvieux avec des précipitations s'étendant sur 9 mois. Ces précipitations moyennes mensuelles varient de $55 \mathrm{~mm}$ en décembre à $800 \mathrm{~mm}$ en août avec une moyenne annuelle de $360,83 \mathrm{~mm}$. L'humidité varie entre $62,3 \%$ en mai à $88,6 \%$ en août avec une moyenne annuelle de $78,3 \%$. L'évaporation varie entre $32,5 \%$ en août à $66,6 \%$ en mars avec une moyenne annuelle de 50,6\%. L'insolation moyenne mensuelle varie entre $151 \%$ en avril à $69 \%$ en septembre avec une moyenne annuelle de 109,6\%. Le vent dominant est porteur de mousson avec seulement $48 \%$ de calme à Douala (Folack et al., 1999). La majeure partie de Douala a une couverture géologique constituée par formations du socle essentiellement représentées par des gneiss-embredistes à biotite et secondairement par des anatexies, des granites syntectoniques non circonscrits et circonscrits. Les sols dérivés de ces roches sont très variés: sols ferralitiques rouges dérivant de roches métamorphiques ou de basalte et sols bruns dérivés de basalte (Folack et al., 1999). La végétation de Douala est essentiellement constituée par une forêt dense sempervirente atlantique de basse et moyenne altitude à Lophira alata dans la zone littorale et à Fabacées en moyenne altitude (Folack et al., 1999). L'étude s'est déroulée à la rivière Kambo où trois stations d'échantillonnage ont été choisies selon le gradient longitudinal du cours d'eau. La rivière couvre les quartiers Yassa, station 1 (amont); Arir, station 2 (milieu) et Cogefar, station 3 (aval). Les activités anthropiques principales de la région sont l'agriculture, l'élevage porcin et surtout les constructions anarchiques pour l'habitation. Les déchets ménagers sont déversés directement dans le lit du cours d'eau. II existe aussi plusieurs dépôts de déchets des brasseries connus sous le nom de " drèche » destinés à la commercialisation pour la nutrition des porcs. Les eaux de lessivage entrainent cette " drèche » dans le cours d'eau du sous bassin. Une autre activité de la région est la pêche aux poissons pratiquée la plupart du temps par les adolescents. Ces eaux sont aussi des lieux de loisirs où les jeunes viennent nager.

Méthodologie : Deux transects linéaires de 50 m chacun ont été effectués à chaque station, pour les inventaires floristiques selon le gradient longitudinal du cours d'eau. Des parcelles de $1 \mathrm{~m}^{2}$ pour les herbacées et $5 \mathrm{~m}^{2}$ pour ligneux, distantes de $5 \mathrm{~m}$ ont été délimitées de part et d'autre de chaque transect pour les relevés floristiques. A l'analyse floristique a été associée des facteurs physico-chimiques mesurés pour l'établissement des corrélations probables. Le recouvrement total de la végétation, la répartition hydrophytes/hélophytes, la largeur moyenne du lit mouillé, la longueur du transect, la superficie moyenne en eau et la superficie moyenne hors eau ont été évalués. L'abondance-dominance de chaque relevé a été appréciée suivant l'échelle de BraunBlanquet (1964) et l'indice de Massens (1997) :

$+=$ recouvrement $<1 \%$ correspondant à un recouvrement moyen de 0,5 ;

1 = recouvrement 1 à $5 \%$ correspondant à un recouvrement moyen de 3 ;

2 = recouvrement 5 à $25 \%$ correspondant à un recouvrement moyen de 15 ;

$3=$ recouvrement 25 à $50 \%$ correspondant à un recouvrement moyen de 37,5 ;

4 = recouvrement 50 à $75 \%$ correspondant à un recouvrement moyen de 62,5 ;

$5=$ recouvrement 75 à $100 \%$ correspondant à un recouvrement moyen de 87,5 .

La sociabilité des espèces suivant l'échelle d'Adam (1964) a été ensuite donnée : 5 = peuplements denses ; 4 $=$ petites colonies ; $3=$ petits groupes $; 2=$ groupes restreints ; 1 = isolé. Les indices d'abondance-dominance permettent de calculer pour chaque espèce son recouvrement moyen (RM) qui est le pourcentage d'occupation moyen de cette espèce dans un milieu donné $: \mathrm{RMi}=(\mathrm{Ri} /$ Nombre total de relevés $) \times 100$ où $\mathrm{Ri}$ est le recouvrement de cette espèce dans le milieu. L'indice de présence $(\mathrm{Pi})$ correspond au recouvrement moyen de l'espèce i sur le recouvrement total des individus $\left(\mathrm{Pi}=\mathrm{RMi} / \sum \mathrm{RM}\right)$. L'indice de Shannon-Weaver $\left(H^{\prime}\right)$ indique la diversité ou la richesse spécifique du milieu, il est donc déterminé par la relation suivante :

$$
H^{\prime}=-\sum \mathrm{Pi} \times \log _{2} \mathrm{Pi}
$$


L'indice de régularité ou "Evennes indice" ou équitabilité de Pielou est : $\mathrm{R}=\mathrm{H}^{\prime} / \mathrm{H}^{\prime} \max , \mathrm{H}^{\prime} \max$ étant la diversité maximale $\left(\log _{2} S\right)$ où $S$ est le nombre d'espèces (Dajoz, 1985 ; Ngueguim et al., 2010). La régularité permet d'appréhender le désordre relatif de la population. Elle tend vers 0 quand la quasi-totalité des effectifs est concentrée sur une seule espèce. Elle est de 1 lorsque toutes les espèces ont la même abondance. Une régularité faible représente la grande importance de quelques espèces dominantes (Dajoz, 1985). De même, le coefficient de similitude de Sorensen $(Q)$ met l'accent remarquable sur la présence conjointe de deux espèces au même endroit : $Q=[2 a /(2 a+b+c)]$ avec $0<Q<1$, $a$ $=$ nombre d'espèces communes aux deux milieux; $b=$ nombre d'espèces présentes dans le milieu $A$ et absentes dans le milieu $B, C=$ nombre d'espèces présentes dans le milieu $B$ et absentes dans le milieu $A$.
Tableau des groupements: Dans les tableaux représentant les cortèges floristiques des groupements, il est remplacé les numéros de relevé de terrain par des numéros qui se suivent de manière progressive du premier au dernier groupement étudié. Dans le tableau phytosociologique de chaque groupement, le numéro de relevé, le recouvrement, le nombre d'espèces par relevé, la fréquence de chaque espèce et la classe de fréquence sont notés. La classe de fréquence représente les espèces présentes dans : 0 à $20 \%$ des relevés, classe I ; 20 à $40 \%$ des relevés, classe $11 ; 40$ à $60 \%$ des relevés, classe III ; 60 à $80 \%$ des relevés, classe IV ; 80 à $100 \%$ des relevés, classe V. Le coefficient de variation des espèces des groupements est donné selon la formule (Thiam, 1984) :

$\mathrm{Cv}=\frac{S x}{\bar{X}}($ avec $\mathrm{Cv}=$ coefficient de variation, $\mathrm{Sx}=$ Variance des espèces du groupement et

$X=$ moyenne des espèces du groupement) ;

$S x=\sqrt{\frac{\sum x^{2}-\frac{(\Sigma x)^{2}}{n}}{n-1}}$ (avec $S x=$ variance, $\mathrm{X}=$ nombre d'espèces de chaque relevé et
$\mathrm{n}=$ effectif total du groupement).

Méthodes d'étude des facteurs écologiques: La granulométrie est la proportion des éléments du sol classés par catégorie de grosseur (vase, sable, gravier et galet). Elle indique la texture du sol. De celle-ci dépend la valeur agricole du sol, son pouvoir de rétention de l'eau. Le pH désigne l'acidité du sol. Sa valeur reflète approximativement la quantité d'ions $\mathrm{H}^{+}$libres se trouvant dans la solution du sol (Thiam, 1984). Elle s'exprime par le $\mathrm{pH}$ de la suspension obtenue en délayant un poids déterminé de terre dans un certain volume d'eau distillée (rapport sol/eau $=1 / 2,5)$. L'échelle $\mathrm{du} \mathrm{pH}$ qui a été adoptée est la suivante : $4,5=$ extrêmement acide ; 4,6 à $5=$ très fortement acide ; 5,1 à $5,5=$ fortement acide $; 5,6$ à 6 = moyennement acide ; 6,1 à $6,5=$ faiblement acide ; 6,6 à $7,3=$ neutre ; 7,4 à $7,8=$ légèrement alcalin ; 7,9 à $8,4=$ moyennement alcalin ; 8,5 à $9=$ fortement alcalin ; $9,1=$ très fortement alcalin .

La conductivité donne des renseignements sur la salinité du sol et de l'eau. II est admis que la salinité d'un sol s'exprime en conductivité spécifique à $25^{\circ} \mathrm{C}$ de la solution obtenue à partir du sol (Durand, 1983). Pour cela l'extrait 1/10 (utilisation d'une quantité d'eau représentant 10 fois, le poids du sol) a été utilisé. Pour les mesures de conductivité, l'échelle utilisée est celle de Durand (1983) (Tableau 1).

Tableau 1 : Échelle de conductivité.

\begin{tabular}{|l|l|l|l|l|l|}
\hline Degré de salinité & Non salin & $\begin{array}{l}\text { Légèrement } \\
\text { salin }\end{array}$ & Salin & Très salin & Extrêmement salin \\
\hline $\begin{array}{l}\mathrm{CE} 25^{\circ} \mathrm{C} \text { extrait } \\
1 / 10 \mathrm{en} \mu \mathrm{S} / \mathrm{cm}\end{array}$ & $0-250$ & $250-500$ & $500-1000$ & $1000-2000$ & $>2000$ \\
\hline
\end{tabular}

La température ambiante des différents relevés a été mesurée entre $8 \mathrm{~h}$ et $11 \mathrm{~h}$. la profondeur de l'eau a été mesurée in situ à trois endroits (aux deux rives et au milieu) à l'aide d'un bâton gradué.
Le débit de l'eau a été mesuré par une corde munie d'un flotteur qui a été lancée dans l'eau, suivant une distance de $10 \mathrm{~m}$. le chronomètre a permis de prendre le 
temps et la formule suivante a permis de calculer le débit de l'eau:

$V=\frac{d}{t}(V=$ vitesse en $\mathrm{m} / \mathrm{s}, \mathrm{d}=$ temps en seconde et $\mathrm{t}=$ distance parcourue en $\mathrm{m}$ )

Analyses statistiques : Les données ont été traitées par Microsoft Office Excel 2007. Une ACP (analyse en composantes principales) a été effectué pour la

\section{RÉSULTATS}

Paramètres physico-chimiques: Le $\mathrm{pH}$ est un indicateur de la quantité et de la nature des ions minéraux en solution. Ce paramètre permet d'estimer le degré d'agressivité d'une zone et son aptitude à la vie animale supérieure (Groga, 2012). Dans les sites, le répartition spatio-temporelle des paramètres abiotiques grâce au logiciel $R$ d'analyses statistiques version R 386 3.0.1. Une AFC (analyse factorielle par correspondance) a été effectué pour la répartition des principaux groupements des macrophytes en relation avec le milieu par le logiciel XLSTAT 2014 ainsi que des dendodogrammes.

potentiel hydrogène ne montre pas d'importantes variations. Ces variations dépendent essentiellement des processus chimiques mais aussi biologiques. Le pH du sol montre qu'il est légèrement acide dans les stations 1 et 2, et neutre dans la station 3 (Tableau 2).

Tableau 2. Paramètres physico-chimiques des différentes stations.

\begin{tabular}{|c|c|c|c|}
\hline \multirow{2}{*}{ Paramètres } & \multicolumn{3}{|c|}{ Stations } \\
\cline { 2 - 4 } & $\mathbf{1}$ & $\mathbf{2}$ & $\mathbf{3}$ \\
\hline $\mathrm{Ta}\left({ }^{\circ} \mathrm{C}\right)$ & $28,65 \pm 0,30$ & $28,41 \pm 0,32$ & $28,06 \pm 0,76$ \\
\hline $\mathrm{Ts}\left({ }^{\circ} \mathrm{C}\right)$ & $26,50 \pm 0,02$ & $27,50 \pm 0,03$ & $28,10 \pm 0,01$ \\
\hline Prof. de l'eau $(\mathrm{cm})$ & $50,00 \pm 13,00$ & $70,00 \pm 50,00$ & $150,00 \pm 35,00$ \\
\hline Cond. de l'eau $(\mu \mathrm{s} / \mathrm{cm})$ & $57,00 \pm 0,21$ & $49,20 \pm 0,35$ & $63,70 \pm 0,24$ \\
\hline $\mathrm{pH}$ de l'eau & $6,45 \pm 0,02$ & $6,49 \pm 0,03$ & $6,96 \pm 0,01$ \\
\hline Débit (m/s) & $0,20 \pm 0,04$ & $0,15 \pm 0,00$ & $0,06 \pm 0,03$ \\
\hline $\mathrm{pH}$ du sol & $6,45 \pm 0,17$ & $6,46 \pm 0,04$ & $6,83 \pm 0,07$ \\
\hline Cond. du sol $(\mu S / \mathrm{cm})$ & $52,43 \pm 16,50$ & $54,18 \pm 13,89$ & $60,69 \pm 6,11$ \\
\hline Vase $(\%)$ & $84,75 \pm 7,09$ & $92,48 \pm 7,05$ & $90,66 \pm 7,00$ \\
\hline Sable $(\%)$ & $15,25 \pm 7,09$ & $7,51 \pm 7,05$ & $9,33 \pm 7,00$ \\
\hline
\end{tabular}

( $\mathrm{Ta}=$ température ambiante, $\mathrm{Ts}=$ température de l'eau, Prof. = profondeur, Cond. = conductivité).

Typologie des stations : L'examen de l'organisation des 10 variables sur les plans F1 x F2 de l'ACP, représentée sous forme de cercles de corrélations révèle que l'axe factoriel F1 oppose deux ensembles de variables. II est corrélé positivement avec les éléments du premier ensemble contenant des descripteurs très bien corrélés entre eux qui sont le débit de l'eau et la température ambiante. La composante principale F1 définit donc un gradient de courant de l'eau. Ce premier axe factoriel est corrélé négativement aux éléments de minéralisation ( $\mathrm{pH}$ et conductivité du sol et aux températures contribuant fortement à la formation de cette composante principale. L'axe factoriel F2 est corrélé positivement par à la conductivité de l'eau, le sable et corrélé négativement à la vase (Fig. 1). 


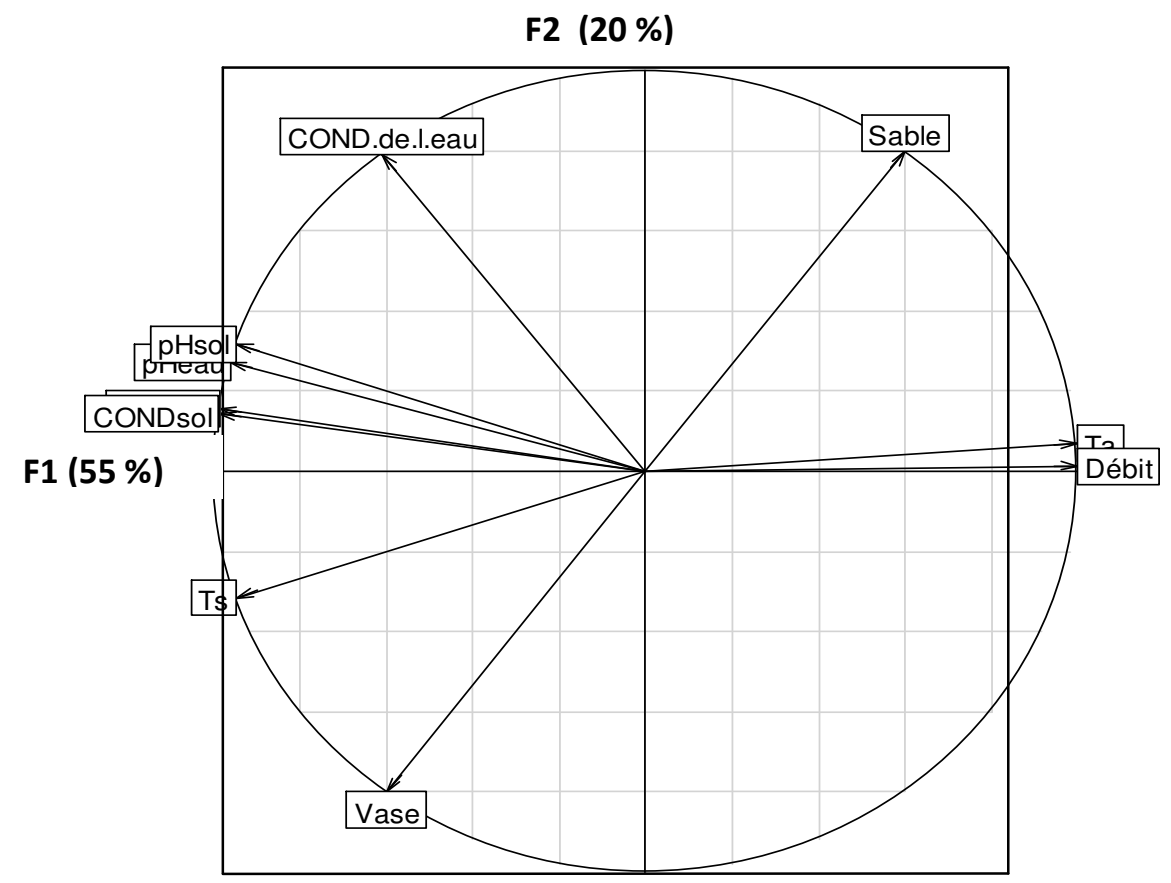

Fig. 1. Cercle de corrélation des 10 variables dans les plans F1 $\times$ F2 de l'ACP : Cond. = conductivité, $p H=$ potentiel d'hydrogène, $\mathrm{Ts}=$ température de l'eau, $\mathrm{Ta}=$ température ambiante, Prof. = profondeur.

Analyse phytosociologique des milieux échantillonnés: Dans ce site, les secteurs biogéographiques sont Yassa (amont), Arir (milieu) et Cogefar (aval). La largeur moyenne du lit mouillé varie de $25 \mathrm{~m}$ (station 2) à $50 \mathrm{~m}$ (station 3) avec une moyenne de $35 \pm 20 \mathrm{~m}$ (Tableau 3). La végétation est à strates herbacées climaciques dans la station 1 et 2 laissant la place à une strate arbustive dans la station 3. Les abords du lit mouillé des stations 1 et 2 sont cultivés. La station 3 (aval) est située dans une vallée mouillée aplatie. Les abords de cette station présentent une pente abrupte d'environ $90^{\circ}$, justifiant l'abandon de cette zone par les populations concernées. Le recouvrement moyen du lit mouillé dans ce milieu est plus élevé à cause de sa situation géographique. Elle est influencée par les activités anthropiques notamment la construction des habitats. L'installation des personnes dans ce milieu entraine une dégradation du milieu. Ces personnes en remblant leurs terrains drainent les eaux en creusant. Cette pratique entraine un agrandissement du cours d'eau influençant négativement la végétation par la régression des macrophytes aquatiques. D'autre part, les voiries non revêtues qui y sont créées contribuent davantage à dégrader la végétation.

Tableau 3 : Analyse phytosociologique des milieux échantillonnés dans les stations.

\begin{tabular}{|l|c|c|c|}
\hline Critères & \multicolumn{3}{|c|}{ Stations } \\
\hline Code transect & 1 (Yassa) & 2 (Arir) & 3 (Cogefar) \\
\hline Largeur moyenne du lit mouillé $(\mathrm{m})$ & 30,00 & 25,00 & 50,00 \\
\hline Superficie moyenne en eau (\%) & 37,00 & 46,57 & 22,50 \\
\hline Superficie moyenne hors eau (\%) & 63,00 & 53,43 & 77,50 \\
\hline Recouvrement de la végétation totale (\%) & 97,30 & 75,61 & 80,00 \\
\hline Recouvrement des hydrophytes (\%) & 2,30 & 12,76 & 14,00 \\
\hline Recouvrement des hélophytes (\%) & 97,70 & 87,24 & 86,00 \\
\hline Type de milieu & Ruisseaux & Rivière & Rivière \\
\hline
\end{tabular}



Kambo à Douala (Cameroun)

Richesse spécifique: La zone d'étude apparait diversifiée avec une richesse spécifique élevée comprenant 42 espèces distribuées dans 25 familles. La famille la plus diversifiée est celle des Poacées (5 espèces) notamment les Acanthacées: Asystasia gangetica (L.) T. Anderson, A. vogeliana Benth.; Amaranthacées: Amaranthus viridis L. (green amaranth); Apocynacées: Alstonia boonei De Wild. (emien) ; Aracées : Cyrtosperma senegalense (Schott.) Engl. (macabo de marécage), Xanthosoma sagittifolium (L.) Schott (macabo); Arécacées: Eleais guineensis Jacq. (palmier à huile), Raphia sp.; Astéracées: Ageratum conizoides L., Chromolaena odorata (L.) R. M. King \& H. Rob. (herbe de Laos), Eclipta prostrata (L.) L. (mahakanni); Vernonia sp. ; Commélinacées: Commelina benghalensis L. (benghal dayflower), Palisota hirsuta (Thunb.) K. Schum., Convolvulacées : Ipomea batatas (L.) Lam. (patate douce), I. involucrata P. Beauv. (ipomée); Costacées: Costus afer Ker.-Gawl.; Cypéracées: Cyperus difformis L. (umbrella sedge), C. haspan L., Entada africana Guill. \& Perr. (légume); Euphorbiacées: Alchornea cordifolia Müll. Arg., Phyllanthus amarus Schum. \& Thonn. (grain en bas de feuille), Uapaca sp.; Mélastomatacées: Dissotis prostrata (Thonn.) Hook. F. , Dissotis sp. ; Onagracées: Ludwigia abyssinica A. Rich., L. palustris; Poacées:
Echinochloa colona (L.) Link (sawa millet), E. pyramidalis (Lam.) Hitchc., Leersia hexandra Sw., Panicum repens L., Setaria verticillata (L.) P. Beauv. (bristly foxtail); Pontedériacées: Eicchornia crassipes (Mart.) Solms (jacinthe d'eau). Ensuite viennent les Convolvulacées (3 espèces), les Asteracées (3 espèces), les Euphorbiacées (3 espèces) et les Arecacées (3 espèces) avec une proportion de $6,25 \%$. La physionomie générale de la végétation de cette zone humide montre que l'amont est moins diversifié que l'aval.

Indices de diversité : L'indice de diversité de ShannonWeaver concernant les herbacées est décroissant de la station 1 à la station 3 , respectivement de 2,96 à 1,76. La station 3 possède un autre indice de diversité concernant les ligneuses qui est de 1,47. La régularité est décroissante de la station $1(0,67)$ à la station $3(0,40)$ pour les herbacées. Cette régularité est de 0,46 pour les ligneux à la station 3. L'indice de diversité maximal des herbacées varie de 4,52 (station 2) à 4,39 (stations 1 et 3). Celui des ligneux est de 3,16 (Tableau 4). La diversité associée à la régularité montre dans l'ensemble un gradient de distribution des macrophytes: l'amont présente une diversité plus importante que l'aval. Cette perturbation est marquée par les activités observées sur le terrain.

Tableau 4 : Indices de diversité des stations du site.

\begin{tabular}{|c|c|c|c|c|c|}
\hline \multicolumn{3}{|c|}{ Site d'étude } & \multicolumn{3}{|c|}{ Indices de diversité } \\
\hline \multicolumn{3}{|c|}{ 然 } & $\mathrm{H}^{\prime}$ & $\mathbf{R}$ & $H^{\prime} \max$ \\
\hline \multirow{4}{*}{ Station } & 1 & Herbacées & 2,96 & 0,67 & 4,39 \\
\hline & 2 & Herbacées & 2,41 & 0,53 & 4,52 \\
\hline & \multirow[t]{2}{*}{3} & Herbacées & 1,76 & 0,40 & 4,39 \\
\hline & & Ligneuses & 1,47 & 0,46 & 3,16 \\
\hline
\end{tabular}

Indice de similitude: La richesse spécifique est maximale à la station 3 avec 32 espèces et minimale à la station 1 avec 21 espèces. La station 1 possède 7 espèces spécifiques, la station 2 possède 4 espèces spécifiques et la station 3 possède 16 espèces spécifiques. L'indice de similitude est élevé entre les stations 1 et 2 , et d'une valeur de 0,54. II est minimal entre les stations 1 et 3 , et d'une valeur de 0,33 . Le nombre d'espèces communes aux trois stations est de 7 (Tableau 5).

Tableau 5. Indices de similitude entre les différentes stations du site.

\begin{tabular}{|c|c|}
\hline Stations & Indices de similitude \\
\hline $1-2$ & 0,54 \\
\hline $1-3$ & 0,33 \\
\hline $2-3$ & 0,47 \\
\hline
\end{tabular}

Les groupements végétaux en relation avec le milieu : Au total 128 relevés de $1 \mathrm{~m}^{2}$ et $5 \mathrm{~m}^{2}$ ont été confectionnés. La végétation du site étudié s'organise en groupements végétaux dans lesquels cohabitent des espèces qui y trouvent des conditions favorables. Les facteurs écologiques prépondérants ne sont pas les 

Kambo à Douala (Cameroun)

mêmes pour toutes les espèces réunies. Le groupement végétal résulte de la juxtaposition de groupes d'espèces liés chacun à la variation des facteurs écologiques.

Principaux groupements de la station 1 (Yassa) : Le groupement 1 ( 6 relevés) possède 10 espèces : Leersia hexandra, Mimosa pudica, Ipomea batatas, Asystasia vogeliana, Commelina benghalensis, Ipomea involucrata, Asystasia gangetica, Amaranthus viridis, Striga hermonthica. et Ludwigia abyssinica. II s'étend sur une longueur de $13 \mathrm{~m}$ environ. Leersia hexandra apparait caractéristique de ce milieu. Mimosa pudica et Ipomea batatas sont les espèces compagnes les plus abondantes apparaissant dans 4 relevés sur 6 . Quatre espèces sont exclusives à ce groupement: Asystasia gangetica, Amaranthus viridis, Striga hermonthica et Ludwigia abyssinica. Parmi ces espèces, Striga hermonthica est accidentelle au groupement. La moyenne d'espèces par relevé est de 4,16. Le coefficient de variation des espèces dans ce groupement est de $33 \%$.

Le groupement 2 (12 relevés) possède 8 espèces: Leersia hexandra, Echinochloa pyramidalis, Asystasia vogeliana, Mimosa pudica, Ipomea batatas, Echinochloa colona, Ipomea involucrata et Amaranthus viridis. Leersia hexandra et Echinochloa pyramidalis y sont caractéristiques. Ce groupement a une longueur de $31 \mathrm{~m}$ environ. Mimosa pudica et Asystasia vogeliana sont compagnes et apparaissent dans la majorité des relevés. Dans ce groupement, aucune espèce exclusive n'est présente. La moyenne du nombre d'espèces par relevé est de 3,83. Le coefficient de variation des espèces dans ce groupement est de $29 \%$.

Le groupement 3 (12 relevés) est à Pteridium aquilinum avec une longueur de $31 \mathrm{~m}$ et constitué de 10 espèces : Pteridium aquilinum, Setaria verticillata, Asystasia vogeliana, Commelina benghalensis, Cyrtosperma senegalense, Leersia hexandra, Echinochloa pyramidalis, Oxalis barrelieri, Ageratum conyzoides et Borreria monticola. Pteridium aquilinum est l'espèce caractéristique de ce groupement. Cette espèce est suivie de Setaria verticillata qui est compagne. Dans ce groupement 3 espèces sont exclusives : Oxalis barrelieri, Ageratum conyzoides et Borreria monticola. La moyenne des espèces par relevé est de 3,5. Le coefficient de variation des espèces de ce groupement est de $35 \%$. Le groupement 4 (12 relevés) est long de $31 \mathrm{~m}$, avec 9 espèces : Echinochloa pyramidalis, Asystasia vogeliana, Eicchornia crassipes, Mimosa pudica, Costus afer, Commelina benghalensis, Chromolaena odorata, Eclipta prostrata et Palisota hirsuta. Echinochloa pyramidalis et Asystasia vogeliana sont caractéristiques de ce groupement. L'espèce compagne est Eicchornia crassipes. Ce groupement a 3 espèces exclusives: Chromolaena odorata, Eclipta prostrata et Palisota hirsuta. Le nombre moyen d'espèces par relevé est de 2,66 et le coefficient de variation des espèces de ce groupement est de $29 \%$.

Le groupement 5 (12 relevés) a $31 \mathrm{~m}$ de long, avec 9 espèces : Leersia hexandra, Asystasia vogeliana, Ipomea batatas, Asystasia gangetica, Commelina benghalensis, Ageratum conyzoides, Amaranthus viridis et Ludwigia abyssinica. Asystasia vogeliana apparait majoritairement dans les relevés avec une présence de $67 \%$. Ce groupement a 4 espèces exclusives : Commelina benghalensis, Ageratum conyzoides, Amaranthus viridis et Ludwigia abyssinica. Le nombre moyen d'espèces par relevé est de 2,9. Le coefficient de variation des espèces de ce groupement est de $44 \%$.

Principaux groupements de la station 2 (Arir) : Le groupement 6 (10 relevés) est d'une longueur de $25 \mathrm{~m}$ avec 8 espèces : Leersia hexandra, Amaranthus viridis, Ludwigia abyssinica, Dissotis prostrata, Costus afer, Cyperus haspan, Pteridium aquilinum et Commelina benghalensis. Il est caractérisé par une espèce, Leersia hexandra avec une abondance-dominance maximale dans tous les relevés. Une seule espèce est exclusive au groupement et apparait dans le premier relevé avec un recouvrement $<1 \%$. II s'agit de Commelina benghalensis. Le nombre moyen d'espèces par relevé est de 2,9 . Le coefficient de variation des espèces de ce groupement est de $44 \%$.

Le groupement 7 (16 relevés) a une longueur de $43 \mathrm{~m}$, avec 16 espèces: Pteridium aquilinum, Costus afer, Setaria verticillata, Cyrtosperma senegalense, Leersia hexandra, Dissotis sp., Ludwigia abyssinica, Chromolaena odorata, Commelina benghalensis, Borreria verticillata, Dissotis prostrata, Xanthosoma sagittifolium, Ageratum conyzoides, Oxalis barrelieri, Phyllanthus amarus et Vernonia sp. Ce groupement est caractérisé par Pteridium aquilinum qui apparait dans tous les relevés. Costus afer est présente dans près de la moitié des relevés. Ce groupement a 6 espèces exclusives : Dissotis prostrata, Xanthosoma sagittifolium, Ageratum conyzoides, Oxalis barrelieri, Phyllanthus amarus et Vernonia sp. Le nombre moyen d'espèces par relevé est de 3,28. Le coefficient de variation des espèces de ce groupement est de $46 \%$.

Le groupement 8 ( 14 relevés) est de longueur $37 \mathrm{~m}$, avec 12 espèces: Leersia hexandra, Pteridium aquilinum, Cyrtosperma senegalense, Cyperus difformis, Phyllanthus amarus, Chromolaena odorata, Commelina benghalensis, Asystasia vogeliana, Oxalis barrelieri, Ageratum conyzoides, Amaranthus viridis et Asystasia 
gangetica. II est caractérisé par Leersia hexandra qui se retrouve majoritairement dans tous les relevés. L'espèce compagne majoritaire de ce groupement est Pteridium aquilinum, avec une présence de $79 \%$. Ce groupement a 4 espèces exclusives : Oxalis barrelieri, ageratum conyzoides, Amaranthus viridis et Asystasia gangetica. Le nombre moyen d'espèces par relevés est de 3,83 . Le coefficient de variation des espèces de ce groupement est de $50 \%$.

Principaux groupements de la station 3 (Cogefar) : La longueur du groupement 9 (10 relevés) est de $25 \mathrm{~m}$ avec 12 espèces: Cyrtosperma senegalense, Panicum repens, Alchornea cordifolia, Pteridium aquilinum, Hallea stipulosa, Costus afer, Chromolaena odorata, Raphia sp., Dissotis prostrata, Phyllanthus amarus, Eleais guineensis et Ludwigia palustris. Ce groupement a 3 espèces caractéristiques: deux herbacées (Panicum repens et Cyrtosperma senegalense) et une ligneuse (Alchornea cordifolia). Pteridium aquilinum dans ce groupement apparait comme compagne avec une fréquence de $70 \%$. Deux espèces sont exclusives au groupement, Ludwigia palustris et Eleais guineensis. Le nombre moyen d'espèces par relevé est de 3,83 . Le coefficient de variation des espèces de groupement est de $50 \%$.

Le groupement 10 (10 relevés) a $25 \mathrm{~m}$ de long, avec 13 espèces: Panicum repens, Alchornea cordifolia, Pteridium aquilinum, Cyperus difformis, Entada africana, Cyrtosperma senegalense, Ludwigia palustris, Dissotis prostrata, Eleais guineensis, Alstonia boonei, Chromolaena odorata, Phyllanthus amarus et Vernonia sp. Il est caractérisé par une espèce herbacée, Panicum repens et une ligneuse, Hallea stipulosa . Pteridium aquilinum se fait aussi remarquer par sa forte présence comme compagne dans ce groupement. Deux espèces exclusives sont présentes: Phyllanthus amarus et
Vernonia sp. Le nombre moyen d'espèces par relevé est de 3,71. Le coefficient de variation des espèces de ce groupement est de $42 \%$.

Le groupement 11 (10 relevés) a une longueur de $25 \mathrm{~m}$, avec 13 espèces : Cyrtosperma senegalense, Pteridium aquilinum, Alchornea cordifolia, Alstonia boonei, Hallea stipulosa, Leersia hexandra, Dissotis prostrata, Echinochloa pyramidalis, Uacapa sp., Ludwigia palustris, Aframomum sp. et Musanga cecropioides. Trois espèces sont caractéristiques à savoir: Cyrtosperma senegalense, Pteridium aquilinum comme herbacées et une ligneuse qui est Alchornea cordifolia. Alstonia boonei apparait comme l'espèce la plus compagne dans ce groupement. Quatre espèces y sont exclusives : Ludwigia palustris, Aframomum sp. et Musanga cecropioides. Le nombre moyen d'espèces par relevé est de 5,7 . Le coefficient de variation des espèces de ce groupement est de $63 \%$.

Rapprochement des principaux groupements en fonction des paramètres physico-chimiques: Le dendogramme de rapprochement des groupements en fonction des paramètres physico-chimiques montre 3 groupes (Fig. 2) :

le groupe 1: formé des groupements 1 et 3 situés en amont. Cet ensemble est constitué par un sol vaseux ( $78 \%$ de vase), légèrement acide $(\mathrm{pH}=6,48)$ et non salin (Conductivité $=48 \mu \mathrm{S} / \mathrm{cm}$ ) ;

le groupe II: formé des groupements 2 et 6 situés respectivement en amont et au milieu, constitués par un sol très vaseux ( $93 \%$ de vase), neutre $(\mathrm{pH}=6,62)$ et non salin (conductivité $=46 \mu \mathrm{S} / \mathrm{cm}$ ) ;

le groupe III : formé des groupements 4, 5, 7,8, 9,10 et 11 situés respectivement en amont, au milieu et en aval, constitués par un sol très vaseux $(90,30 \%$ de vase), neutre $(\mathrm{pH}=6,6)$ et non salin (conductivité $=60,17$ $\mu \mathrm{S} / \mathrm{cm})$. 


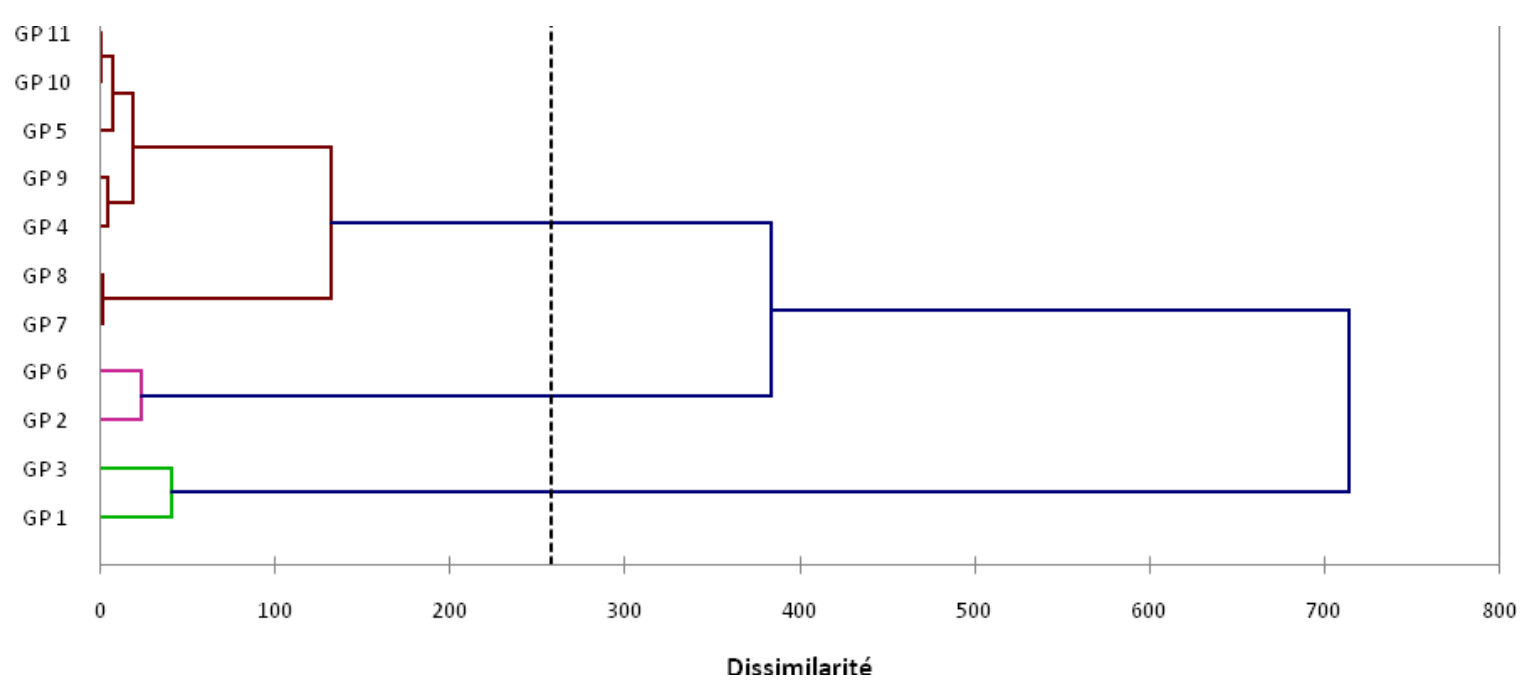

Fig. 2 : Dendogramme de rapprochement des groupements en fonction des paramètres Physico- chimiques.

Variabilité spatiale des espèces en fonction des principaux groupements: Les axes $1(50,98 \%$ d'inertie) et 2 (17,85\% d'inertie) du plan 1-2 de l'analyse factorielle des correspondances (AFC) individualisent quatre groupes (Fig. 3). Les groupes I, II, et III constitués d'espèces exclusives et le groupe IV constitué d'espèces communes à tous les groupements. Le groupe I comprenant les groupements $1,2,3,4,5,6$ et 8 provenant des stations 1 et 2 . Le groupe II comprenant les groupements 9,10 , et 11 provenant de la station 3 . Le groupe III comprenant le groupement 7 provenant de la station 2. Le groupe IV constitué des espèces appartenant à tous les groupements. Les espèces associées au groupe I sont: Palisota hirsuta, Eicchornia crassipes, Eclipta prostrata, Echinochloa colona, Ipomea involucrata, Ipomea batatas, Striga hermonthica,
Asystasia vogeliana, Amaranthus viridis, Asystasia gangetica, Commelina benghalensis, Leersia hexandra, Borreria monticola, Setaria barbata, Echinochloa pyramidalis, Mimosa pudica et Cyperus haspan. Les espèces associées au groupe II sont: Panicum repens, Alchornea cordifolia, Raphia sp., Eleais guineensis, Entada africana, Alstonia boonei, Ludwigia palustris et Hallea stipulosa. Les espèces associées au groupe III sont : Borreria verticillata, Setaria verticillata, Xanthosoma sagittifolium et Dissotis sp. Les espèces associées au groupe IV sont : Cyperus difformis, Chromoleana odorata, Costus afer, Dissotis prostrata, Phyllanthus amarus, Pteridium aquilinum, Cyrtosperma senegalense, Ageratum conyzoides, Oxalis barrelieri, Vernonia sp. et Ludwigia abyssinica. 


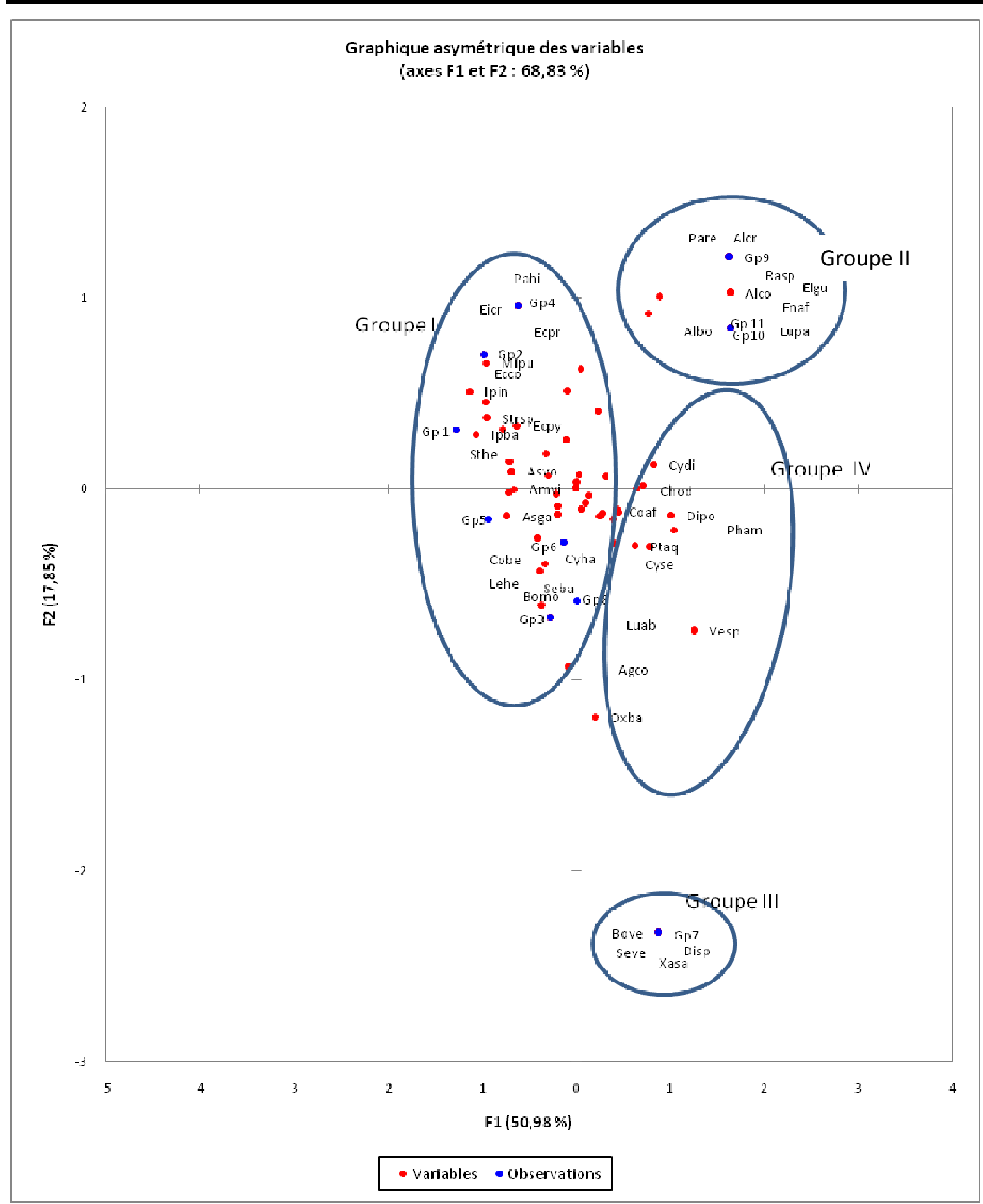

Fig. 3: Analyse factorielle des correspondances réalisée sur la matrice de présence-absence des taxons recensés dans les principaux groupements :

Agco $=$ Ageratum conyzoïdes, Alco $=$ Alchornea cordifolia, Albo $=$ Alstonia boonei, Amvi $=$ Amaranthus viridis, Asga $=$ Asystasia gangetica, Asvo = Asystasia vogeliana,

Bomo $=$ Borreria monticola, Bove $=$ Borreria verticillata,

Chod $=$ Chromolaena odorata, Cobe $=$ Commelina benghalensis, Coaf $=$ Costus afer, Cydi $=$ Cyperus difformis, Cyha $=$ Cyperus haspan, Cyse = Cyrtosperma senegalens, 
Dipo $=$ Dissotis prostrata, Disp $=$ Dissotis sp.,

Ecco $=$ Echinochloa colona, Ecpy $=$ Echinochloa pyramidalis, $\mathrm{Ecpr}=$ Eclipta prostrata, Eicr $=$ Eicchornia crassipens, Elgu $=$ Eleais guineensis, Enaf $=$ Entada africana, Alcr $=$ Hallea stipulosa,

Ipba = Ipomea batatas, Ipin = Ipomea involucrata,

Lehe $=$ Leersia hexandra, Luab = Ludwigia abyssinica, Lupa = Ludwigia palustris,

Mipu $=$ Mimosa pudica,

Oxba $=$ Oxalis barrelieri,

Pahi $=$ Palisota hirsuta, pare $=$ Panicum repens, Pham $=$ Phyllanthus amarus, Ptaq $=$ Pteridium aquilinum,

Rasp = Raphia sp,

Seba $=$ Setaria barbata, Seve $=$ Setaria verticiliata, Sthe $=$ Striga hermonthica,

Xasa $=$ Vesp $=$ Vernonia sp., Xanthosoma sagittifolium .

\section{DISCUSSION}

La variation de la richesse spécifique de la station 1 à la station 3 trouvée est confirmée par Haslam et Wolseley (1987) ayant montré que plus le milieu est dégradé, moins il est diversifié. La sociabilité associée à la préférence d'habitat influence la distribution ou la diversification du milieu. Leersia hexandra qui a une sociabilité au moins égale à 4 , a une préférence pour les sols légèrement acides, non salins et vaseux. Ces deux facteurs limitent la prolifération d'autres espèces dans le milieu (Diop, 2010). La richesse spécifique répertoriée dans le site est très inférieure à celle obtenue par Priso et al. (2012) sur la rivière Kondi, soit 97 espèces. Cette faible valeur serait due au fait que les relevés ne concernait que les macrophytes de la zone de décrue. Cependant, cette richesse spécifique est proche de celle de Hendoux et Cornier (2005), qui ont obtenu 69 espèces vasculaires à l'amont de l'estuaire de la Seine. Les Poacées constituent la famille la plus diversifiée (5 espèces). Priso et al. (2012) vont dans le même sens en montrant aussi que cette famille est la plus diversifiée avec 14 espèces. Sarr et al. (2001) ayant travaillé sur les macrophytes et groupements végétaux aquatiques et amphibies de la basse vallée du Ferlo (Sénégal), ont trouvé 103 espèces végétales ligneuses et herbacées. Ils montrent aussi que lorsque le milieu est soumis à des perturbations d'origine anthropique comme l'eutrophisation, la richesse spécifique tend à diminuer. L'indice de similitude est supérieur à $50 \%$ entre la station 1 et 2 . Ce constat permet de dire que le plus grand nombre d'espèces communes se trouve entre l'amont et le milieu (zone de transition entre l'amont et l'aval). Ces résultats sont proches de ceux obtenus par Priso et al. (2012) qui ont obtenus 68,92\%. Ngueguim et al. (2010) signalent que la similarité des stations est fonction de l'indice de similitude de Sorensen. La similitude des stations herbacées 1 et 2 est plus éloignée de celle de la station 3 qui est une végétation évoluée et constituée de deux strates, herbacée et arbustive. La température est un facteur environnemental important pour la vie contrôlant ainsi l'ensemble des processus biologiques (reproduction, croissance, préférendum thermique) liés à un environnement donné (Aminot, 1983). La vitesse du courant sélectionne les espèces et les types écomorphologiques. Ainsi, dans les eaux courantes la zonation amont-aval des végétaux dépend de la vitesse du courant (Wiegleb, 1984). Ainsi tout en jouant un rôle d'obstacle avec pour effet principal, le ralentissement de l'écoulement, les macrophytes déterminent un piégeage des matières solides transportées (Henri et Amoros, 1996). Ce piégeage favorise la sédimentation des particules contribuant à leur stabilisation pour la protection des berges des rivières, notamment avec l'installation et le développement des hélophytes(Haslam et Woslseley, 1987). La température des eaux de surface est influencée directement par les conditions climatiques (Groga, 2012). Les eaux du cours d'eau de Kambo restent chaudes. Selon Konan et al. (2013) les lagunes sont soumises à des variations saisonnières de la température plus importantes qu'en milieu marin. La plupart des espèces présentes dans les milieux lagunaires supportent donc une large gamme de température. Certaines espèces préfèrent les eaux plus chaudes (Dajoz, 1985). La profondeur et le débit restent faibles dans les stations et sont favorables dans le développement macrophytique. De plus, la colonisation du milieu par les hélophytes suite à l'accumulation des matières plastiques dans l'eau est avantagée. II s'en suit une humification qui facilite l'installation de ligneux caractéristiques d'un stade arbustif (Henry and Amoros, 1996). Des facteurs comme la permanence et la profondeur de l'eau, ses caractéristiques chimiques peuvent être considérées comme essentiels quant à leur influence sur la végétation (Diop, 2010). Les eaux peu chargées en sels, plus ou moins acides (oligotrophes) peuvent héberger des espèces caractéristiques comme Leersia hexandra (Hill 
and Coetzee, 2008). Diop (2010) montre que la compétition interspécifique influence significativement la répartition de la végétation car si une espèce s'installe en premier, sa croissance et sa prolifération peuvent inhiber la croissance d'une autre arrivée secondairement. Tous les groupements de l'axe 1 ne montrent pas un gradient physique ou de minéralisation. Au niveau de l'axe 2 qui oppose le groupe I au groupe II, le taux de vase élevé dans le dernier groupe par rapport au premier en serait la

\section{CONCLUSION}

L'indice de diversité de Shannon-Weaver est variable et décroît de la station 1 à la station 3 , respectivement de 2,96 à 1,76. Les ligneux sont présents uniquement à la station 3 qui est très peu diversifiée avec un indice de 1,47. L'analyse phytosociologique a montré une répartition des groupements végétaux en fonction de l'évolution de la végétation. Les groupements 1, 2, 3, 4, 5, 6 et 8 des stations 1 et 2 sont proches et constitués de strates herbacées alors que les groupements 9,10 et 11

\section{RÉFÉRENCES BIBLIOGRAPHIQUES}

Adam JG. 1964. Contribution à l'étude de la végétation du lac de Guiers (Sénégal). Extrait Bull. IFAN TXXVI Sér. A $n^{\circ} 1,72 p$.

Aminot A. 1983. Manuel des analyses chimiques en milieu marin. Caractéristiques physicochimiques. Centre National de l'exploitation des océans. 39-75.

Barbe J. 1984. Les végétaux aquatiques: Données biologiques et écologiques. Clés de détermination des macrophytes de France. Bull. fr. piscic. $57 n^{\circ}$ spéc. $42 p$.

Bemmo N, Njine T, Nola NM, Ngamga D. 1998. Techniques utilisées au niveau des quartiers péri-urbains pour l'évacuation des eaux usées et excrétas humains : proposition de systèmes appropriés, Yaoundé, Cameroun. $121 \mathrm{p}$.

Braun-Blanquet J. 1964. Pflanzensoziologie. Springer Verlag, Wien. $865 \mathrm{p}$.

Buchez A, Dorigo U, Rimet F. 2010. Surveillance des impacts environnementaux d'effluents aqueux de sites industriels par les Diatomées dulçaquicoles. Étude Record $n^{\circ} 071016 / 1 \mathrm{~A}$. $175 \mathrm{p}$.

Dajoz R. 1985. Précis d'Écologie, 5e ed. Dunod, Paris.

Diop NF. 2010. Intégration de la biodiversité d'eau douce dans le processus de développement en Afrique : Mobilisation de l'information et sites de démonstration. Projet de démonstration du bassin du fleuve Gambie. 48 p. conséquence. II est cependant possible de répartir les groupements en fonction du stade d'évolution de la végétation : les macrophytes ne se répartissent pas au hasard dans un milieu (Priso et al., 2012). Ils présentent une zonation longitudinale selon le cours d'eau. Ainsi, les facteurs phisico-chimiques ne sont pas les seuls responsables de la répartition des principaux groupements (Barbe, 1984).

de la station 3 sont proches et constitués d'une strate arbustive. L'évolution de la végétation est donc nette dans le site d'étude : l'amont et le milieu de la rivière sont à strates herbacées alors que l'aval est à double strate herbacée et arbustive. L'étude écologique réalisée permet de maîtriser les facteurs abiotiques et biotiques qui président à la gestion et la conservation des zones humides côtières, pour le développement durable et le bien être des populations impliquées.

Durand JM. 1983. Les sols irrigables. ACCT / PUF, Paris. $339 \mathrm{p}$.

Dutartre A, Gross F. (1982). Evolution des végétaux aquatiques dans les cours d'eau recalibrés (Exemples pris dans le sud-ouest de la France). In : "Studies on aquatic vascular plants". Symoens JJ, Hooper SS and Compere P (Eds.), Royal Botanical Society of Belgium, Brussels. $394-397$.

Fogwé NZ, Tchotsoua M. 2007. Evaluation géographique de deux décennies de lutte contre les inondations dans la ville de Douala (Cameroun). Rapport, Université de Douala. http://www.infotheque.info/fichiers/JSIR-AUFHanoi07/articles/AJSIR 2-p3 Fogwe.pdf

Folack J, Mbome IL, Bokwe A, Tangang A. 1999. Profil côtier du Cameroun. Projet Grand Écosystème Marin du Golfe de Guinée. Ministère de l'environnement et des fôrets. $73 p$.

Groga N. 2012. Structure, fonctionnement et dynamique du phytoplancton dans le lac de Taabo (Côte d'lvoire). Thèse de Doctorat, université de Toulouse. $224 \mathrm{p}$.

Haslam SM, Wolseley PA. 1987. River plants of Western Europe. The macrophytic vegetation of water courses of the European Economic Community. Cambridge Univ. $512 \mathrm{p}$.

Hendoux F, Cornier T. 2005. Caractérisation phytoécologique des communautés de 
macrophytes de la partie amont de l'estuaire de la seine: identification et hiérarchisation des facteurs écologiques structurant ces communautés et quantification de la productivité en biomasse. Rapport sur les investigations, Centre Régional de Phytosociologie, Conservatoire Botanique National de Bailleul. 61 p.

Henry CP, Amoros C. 1996. Are the banks a source of recolonization after disturbance : an experiment on aquatic vegetation in a former channel of the Rhône River. Hydrobiol. 330 : 151-162.

Hill PM, Coetzee JA. 2008. Integrated control of water hyacinth in Africa. EPPO Bulletin. 38 : 452-457.

Jones RW. 2001. Integrated control of water hyacinth on the Nselemi/Maposa River and lake Nsezi, Kwa Zulu - Natal, South Africa in proceeding of the $2^{\text {nd }}$ Meeting of Global Working Group for the biological and Integrated control of water hyacinthe.

Konan KF, Niamien-Ebrottié JE, Edia OE, Ouattara A, Gourène G. 2013. Composition et variation spatio-saisonnière du peuplement algal des rivières côtières la Cote d'Ivoire. Journal of Applied Biosciences. 66 : 5147-5161

Meva'a AD, Fouda M, Bonglam CZ, Kamwo M. 2010. Analyse spatiale du risque d'inondation dans le bassin versant du Mbanya à Douala, capitale économique du Cameroun. Rapport, NOVATECH, Université de Douala. $10 \mathrm{p}$.

Massens DYB. 1997. Étude phytosociologique de la région de Kikwit (Bandudu, Rép. Dém. Du Congo). Thèse, Université libre de Bruxelles. $398 \mathrm{p}$.

Ngueguim JR, Zapfack L, Youmbi E, Riera B, Onana J, Foahom B, Makombu JG. 2010. Diversité floristique sous canopée en plantation forestière de Mangombe-Edéa (Cameroun). Biotechnology Agronomy Sociology Environment 14 (1) : 167176.

Priso RJ, Dibong SD, Tchinda-Metagne C, Taffouo V, Din N, Amougou A. 2010. Impacts des eaux polluées sur la croissance, les teneurs en chlorophylles et substances organiques dans les feuilles de deux Poaceae. Int. J. Biol. Chem. Sci. 4 (4): 11221129.

Priso RJ, Oum GO, Din N. 2012. Utilisation des macrophytes comme descripteurs de la qualité des eaux de la rivière Kondi dans la ville de Douala (Cameroun-Afrique Centrale). J. Appl. Biosci. 53 : 3797-3811.

Ramade F. 1987. Proposal of ecotoxicological criteria for the assessment of the impact of pollution on environmental quality. Toxicological and Environmental chemistry 13: 189-203.

Saar A., Thiam A., Bâ AT. 2001. Macrophytes et groupements végétaux aquatiques et amphibies de la basse vallée du ferlo (sénégal). African Journal of Science and Technology. 2 (1) : 8997.

Sauberer N, Zulka KP, Abensperg-Traun M, Berg HM, Bieringer $\mathrm{G}$, Milasowsky N, Moser D,

Plutzar C, Pollheimer M, Storch C, Trostl R, Zechmeister H, Grabherr G. 2004. Surrogate taxa for biodiversity in agricultural landscapes of Eastern Austria. Biological Conservation $117: 181-190$

Tatso RP. 2011. Étude exploratoire des techniques de réalisation des cartes d'aléa d'inondation : Cas de la ville de Douala Cameroun. Projet personnel, Université Douala/ENSG UMLV. 16 $p$.

Thiam A. 1984. Contribution á l'étude phytoécologique de la zone de décrus du lac de

Guiers (Sénégal). Thèse de troisième cycle, Institut des Sciences de l'Environnement, Faculté des Sciences et Techniques, Université Cheikh Anta Diop de Dakar. $105 p$.

Wiegleb G. 1984. A study of habitat conditions of the macrophytic vegetation in selected river systems in wastern lower Saxony (Federal Republic of Germany). Aquat. Bot. 18 : 313-352. 